\title{
Comparison of entropy difference in the cooling process
}

\author{
G. Ríos Cruellas \\ Energy, Materials and Systems, Faculty of Science and Technology \\ Carre 2049, P.O. Box 217, 7500 AE Enschede, The Netherlands
}

\begin{abstract}
Summary. - The aim of this study is to create an overview of the various cooling technologies at room temperature. The energy absorbed during the cooling step is validated through an entropy analysis. The comparison involves vaporization, desorption of $\mathrm{H}_{2}$ of metal hydrides, isothermal expansion, magnetocaloric effect and chemical reaction.
\end{abstract}

\section{1. - Introduction}

In this time where technology heads to what is called micro or nano, heat production of small devices becomes an important issue. In order to expel that heat, we need to select technologies with a great cooling potential. Stirling engines, sorption coolers and magnetocaloric refrigerators are some of the technologies already invented for cooling applications.

The cooling cycles we consider in this study, absorb or produce heat at constant temperature (fig. 1a). Against the natural movement of heat, from high to low temperature, the transfer in cooling applications is from low to high temperature. In order to acomplish this, the systems need external work. Since both processes take place at different conditions, ideally another step of the cycle performs work to the system in order to 


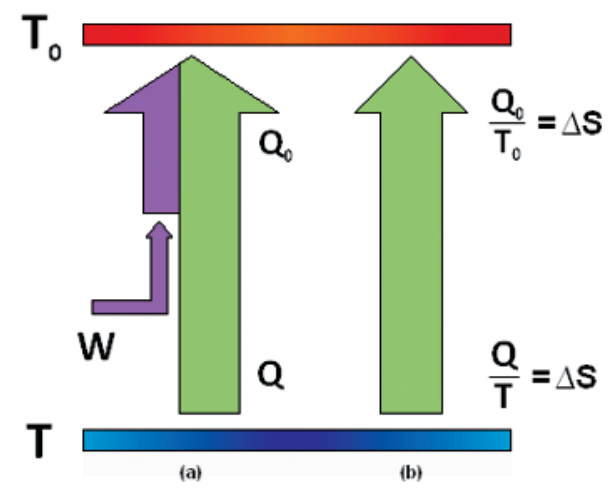

Fig. 1. - Thermodynamic representation of an ideal cooling cycle: (a) energy (b) entropy transfer.

increase or decrease the temperature. That step always keeps a constant property, which is distinctive for each cooling technique.

- Vapour-compression: after evaporation, the liquid increases its temperature in an adiabatic step. After condensing at high temperature, the liquid expands when passing through an isenthalpic valve.

- Absorption of gas in metal hydrides: after absorbing or desorbing the gas, changes in temperature take place with an isosteric process (constant amount of absorbed gas).

- Stirling cycle: after expanding and absorbing heat, the gas increases its temperature at constant volume. The gas expels heat at high temperature, and after that goes back to the initial state through another isochoric step.

- Magnetocaloric refrigeration: magnetic field remains constant when the material undergoes the temperature changes.

- Chemical reaction heat pump: temperature changes take place with constant composition of the working fluid. The temperature increases when it flows through a compressor and decreases with an expansion valve.

The capability to cool at room temperature of various techniques will be validated through an entropy analysis. First, we establish some considerations: our process will take place at constant temperature, we do not have heat loses and it is reversible. If these conditions are satisfied, heat transfer is related to the entropy change of the working substance:

$$
Q=T \Delta S .
$$


TABLE I. - Entropy change of various compounds during evaporation (Source: NIST Database).

\begin{tabular}{cccc}
\hline Compound & $P_{\text {vap }}[\mathrm{bar}]$ & $\Delta s\left[\mathrm{~J} \mathrm{~mol}^{-1} \mathrm{~K}^{-1}\right]$ & $\Delta s / R$ \\
\hline Acetone & 0.155 & 112.64 & 13.55 \\
Ammonia & 6.151 & 73.71 & 8.87 \\
Carbon dioxide & 45.022 & 30.64 & 3.69 \\
Ethanol & 0.032 & 152.25 & 18.31 \\
Methanol & 0.074 & 134.79 & 16.21 \\
R-134a & 4.146 & 68.73 & 8.27 \\
Water & 0.012 & 157.61 & 18.96 \\
\hline
\end{tabular}

This entropy change is the difference between the entropy of the final state and the entropy before the heat transfer. In the case of vaporization, vapour would be the final state and liquid the initial state, both at the boiling temperature. Ideally, this entropy change will be equal when the working substance expels heat at a higher temperature, in the other side of the cycle (fig. 1b):

$$
\Delta S=\frac{Q}{T}=\frac{Q_{0}}{T_{0}}
$$

Ray Radebaugh has already evaluated some cooling technologies for cryogenic applications [1]. In order to compare different technologies, we will make it a dimensionless analysis; all the values are divided by the ideal gas constant $\left(R=8.314 \mathrm{~J} \mathrm{~K}^{-1} \mathrm{~mol}^{-1}\right)$.

\section{2. - Analysis of the various cooling techniques}

$2 \cdot 1$. Vaporization. - In a vapour compression refrigeration cycle, refrigerant is circulated through the system and it undergoes changes in its thermodynamic state. First, the low-pressure refrigerant enters the evaporator where it absorbs heat and vaporizes. After that, it increases its pressure in the compressor and goes to the high-pressure condenser, where it expels heat at high temperature when condensing into liquid. Finally, the high-pressure liquid expands in an expansion valve and returns to the initial state.

The vaporization of a substance is the phase transition from liquid to gas phase. It occurs when the pressure of a system is below the vapour pressure of the liquid at a given temperature. The system must absorb heat to effect the vaporization. In a microscopic view, the heat absorbed is used to overcome the cohesive forces between molecules and separate the atoms to form the vapour.

The saturation pressure of various substances at $10^{\circ} \mathrm{C}$ is shown in table $\mathrm{I}$.

The entropy change $(\Delta s)$ is the difference between the entropy of the liquid and the entropy of the vapour. Water has the highest value, closely followed by ethanol. With this analysis, we could state that water and ethanol have the highest cooling potential for cooling at room temperature by vaporization. 


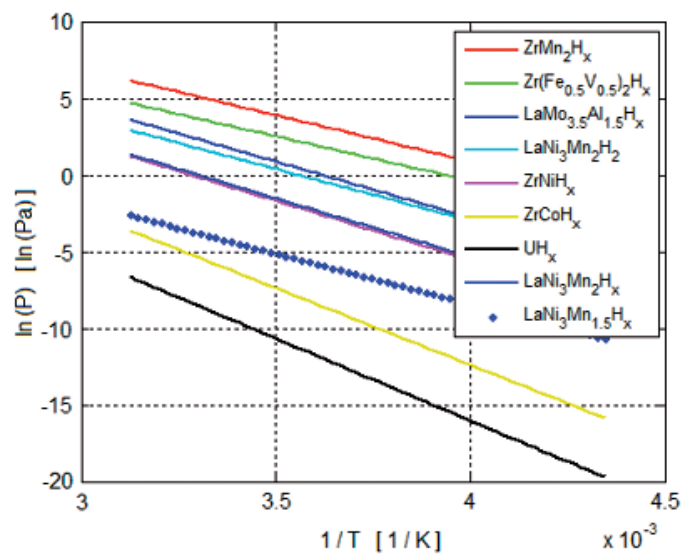

Fig. 2. - Isosteric lines of absorption. The concentrations are between 0.005 and $0.01 g_{\mathrm{H} 2} / g_{\text {absorbent }}[2]$.

When discussing about the evaporation, another important factor is the pressure at which the process takes place. If the difference in entropy is the only criterion, water and ethanol are the best substances for cooling by evaporation at room temperature.

These data refer to specific conditions, which does not mean they are the same at different temperatures. As an interesting fact, the $\Delta s / R$ value for the refrigerant R-134a at $170 \mathrm{~K}$ is 19.01 , much higher than the value at room temperature.

2.2. Desorption of $\mathrm{H}_{2}$ in metal hydrides. - A sorption reactor works in two steps. First, it expels heat when hydrogen is absorbed at high temperature and pressure. In the second step, when temperature and pressure are lower, it absorbs heat while releasing the hydrogen. The sorption coolers comprise two reactors: the first one desorbs the hydrogen at low temperature and it flows to the second one, where it is absorbed at the high temperature.

A very useful way to represent an absorption state is by isosteric lines (fig. 2). They show the pressure as a function of temperature for the same concentration of absorbate $\left(\mathrm{H}_{2}\right)$ in the absorbent $\left(\mathrm{MH}_{x}\right)$.

Clausius-Clapeyron equation describes the relationship between pressure and temperature:

$$
\frac{\partial \ln P}{\partial(1 / T)}=\frac{-T \Delta s}{R} \quad \frac{\Delta s}{R}=-\frac{1}{T} \frac{\partial \ln P}{\partial(1 / T)}
$$

As shown in table II, the highest entropy change happens when $\mathrm{H}_{2}$ absorbs in $\mathrm{UH}_{x}$.

2 3. Isothermal expansion. - In a Stirling cycle, gas expands at constant temperature while it absorbs heat from the ambient. After that reduces its temperature and pressure at constant volume. The gas undergoes an isothermal compression at low temperature 
TABLE II. - Entropy change of the absorption of $\mathrm{H}_{2}$ in various metal hydrides.

\begin{tabular}{cc}
\hline Material & $\Delta s / R$ \\
\hline $\mathrm{ZrMn}_{2} \mathrm{H}_{x}$ & 21.39 \\
$\mathrm{Zr}\left(\mathrm{Fe}_{0.5} \mathrm{~V}_{0.5}\right)_{2} \mathrm{H}_{x}$ & 20.44 \\
$\mathrm{LaMo}_{3.5} \mathrm{Al}_{1.5} \mathrm{H}_{x}$ & 25.77 \\
$\mathrm{LaNi}_{3} \mathrm{Mn}_{2} \mathrm{H}_{2}$ & 23.77 \\
$\mathrm{ZrNiH}_{x}$ & 27.29 \\
$\mathrm{ZrCoH}_{x}$ & 35.39 \\
$\mathrm{UH}_{x}$ & 37.82 \\
$\mathrm{LaNi}_{3} \mathrm{Mn}_{2} \mathrm{H}_{x}$ & 26.96 \\
$\mathrm{LaNi}_{3} \mathrm{Mn}_{1.5} \mathrm{H}_{x}$ & 23.37 \\
\hline
\end{tabular}

as it expels heat to the ambient. Finally, the gas is compressed to the high pressure at constant volume while increasing its temperature and returning to the original state.

Since the internal energy of an ideal gas is a function of temperature, it remains constant during isothermal expansion and compression. Therefore, the heat produced or absorbed has the same value as the work made or taken by the system. Owing to the first law of thermodynamics:

$$
\Delta U=Q+W=0 \quad \rightarrow \quad Q=-W
$$

When compressing an ideal gas volume, the entropy increases since the molecules collide more times per second with each other. Similarly, as the molecules have more room to move, the entropy decreases when expanding an ideal gas. By the definition of work $(W=P d V)$ and the ideal gas law $(P V=n R T)$, we can show that the change in entropy of an isothermal expansion only depends on the pressure ratio:

$$
T \Delta S=Q=-W=n R T \ln \frac{V_{2}}{V_{1}}=n R T \ln \frac{P_{1}}{P_{2}} \quad \rightarrow \quad \frac{\Delta s}{R}=\ln \frac{P_{1}}{P_{2}} .
$$

As shown in fig. 3 , the pressure ratio $\left(P_{1} / P_{2}\right)$ must have values above 20 so that entropy change has values in the same order as in evaporation.

24. Magnetocaloric effect. - When a suitable material undergoes an increase of magnetic field, it needs to expel heat in order to maintain a constant temperature. Likewise, it would reduce its temperature if heat is not applied when the magnetic field decreases.

A decrease in the strength of an externally applied magnetic field allows the magnetic domains of the material to become disoriented. Consequently, the material cools down because the domains absorb the thermal energy to perform their reorientation. Finally, in order to maintain constant temperature, the system increases its entropy absorbing heat from the surroundings. The reverse process occurs in the other side of the cycle, when the working material expels heat at a higher temperature. 


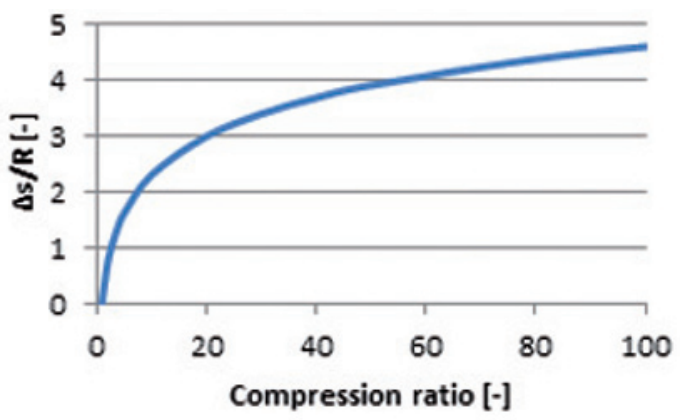

Fig. 3. - Entropy change during isothermal expansion as a function of compression ratio.

As shown in table III, $\mathrm{Gd}_{5} \mathrm{Ge}_{2} \mathrm{Si}_{2}$ has the highest cooling potential at room temperature [3]. However, the change in entropy is much lower than the other techniques analysed above.

Magnetic field constrains the orientation of magnetic dipoles in the refrigerant, so that the material loses some of its internal degrees of freedom. Since this corresponds to lower entropy and heat capacity, magnetocaloric refrigeration would be more useful to attain cryogenic temperatures.

25. Chemical reaction. - Depending on whether chemical reactions expel or absorb heat, they are exothermic or endothermic, respectively. Clearly, we need an endothermic reaction if the purpose is to cool down. For a cooling cycle, the reaction must be reversible in order to expel heat when the reverse reaction takes place. Following the purpose of this study, the reaction has to be feasible at room temperature. Moreover, the reaction enthalpy change should be considerable in order to cool substantially.

In summary, we need a reversible endothermic reaction with a high enthalpy change and high yield at room temperature. Paraldehyde depolymerisation meets all these requirements [4]. In this process, liquid paraldehyde turns into gaseous acetaldehyde while absorbing heat.

TABLE III. - Entropy change of various materials under magnetic induction.

\begin{tabular}{cccc}
\hline Material & $T[\mathrm{~K}]$ & Magnetic induction $[\mathrm{T}]$ & $\Delta s / R$ \\
\hline Gadolinium & 295 & 2 & 0.08 \\
& & 5 & 0.16 \\
$\mathrm{Gd}_{5} \mathrm{Ge}_{2} \mathrm{Si}_{2}$ & 276 & 2 & 1.66 \\
& & 5 & 2.16 \\
$\mathrm{MnFeP}_{0.45} \mathrm{As}_{0.55}$ & 308 & 2 & 0.29 \\
& & 5 & 0.36 \\
\hline
\end{tabular}




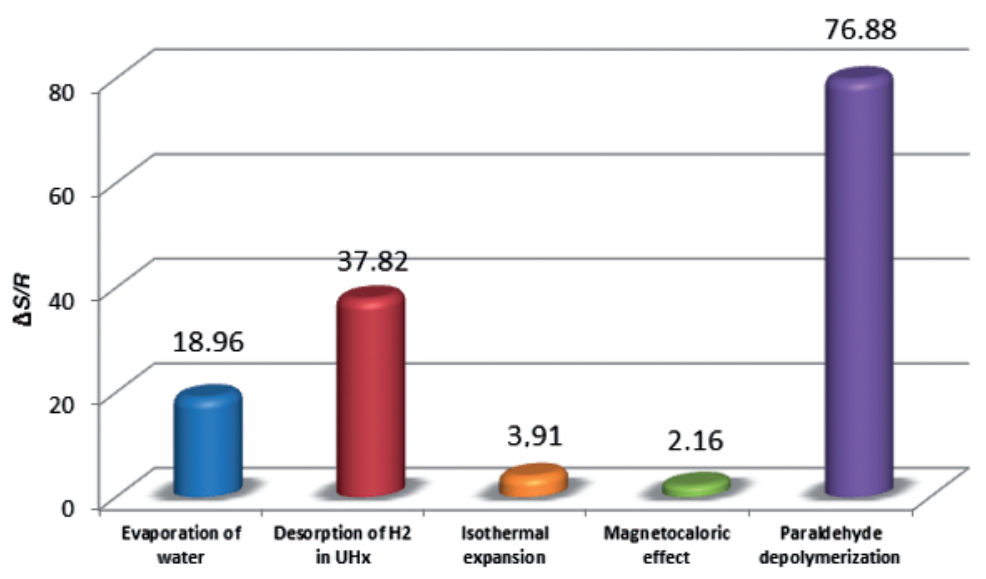

Fig. 4. - Comparison of the entropy change of the analized cooling techniques

We can calculate the entropy change of the process with the difference between entropy of gaseous acetaldehyde and liquid paraldehyde. Entropy increases since we are going from a liquid to a gas. Therefore, the molecules will move faster and collide more times per second with each other. These values are estimates (ASPEN Plus ${ }^{\circledR}$ ) since they do not appear in thermodynamic tables:

$$
\begin{array}{cccc}
S_{A, \text { gas }}\left[\mathrm{J} \mathrm{mol}^{-1} \mathrm{~K}^{-1}\right] & S_{P a, l i q}\left[\mathrm{~J} \mathrm{~mol}^{-1} \mathrm{~K}^{-1}\right] & \Delta s\left[\mathrm{~J} \mathrm{~mol}^{-1} \mathrm{~K}^{-1}\right] & \Delta s / R[-] \\
-90.58 & -910.94 & 639.20 & 76.88
\end{array}
$$

\section{3. - Overview}

We saw that water has the highest cooling potential as a working fluid for evaporation. For desorption, $\mathrm{H}_{2}$ desorbing from $\mathrm{UH}_{x}$ has the highest potential. In the case of isothermal expansion, a compression ratio of 50 seems more than reasonable as an upper limit. $\mathrm{Gd}_{5} \mathrm{Ge}_{2} \mathrm{Si}_{2}$ experienced the highest entropy change when it underwent a 5 tesla magnetic field. Finally, paraldehyde depolymerisation was an example of a chemical reaction for cooling applications at room temperature.

As shown in fig. 4, the entropy change of paraldehyde depolymerisation is above the other values. Evaporation of water and desorption of $\mathrm{H}_{2}$ in $\mathrm{UH}_{x}$ also have a good value; although not as good as the chemical reaction.

Regardless of other factors that affect the performance and velocity of a refrigeration cycle, the chemical reaction is the cooling principle with the highest cooling potential. Nevertheless, in these data we assume that the chemical reaction is complete. If the reactants do not convert totally into products, the heat absorbed will be lower. On the other hand, entropy change is not the only factor to consider if we want to choose the best cooling technique. For example, we are not valuing the velocity of the Stirling engine to perform a complete cycle. It is not discussed whether it would be faster than the others would or not, but rather discusses the cooling potential of isothermal expansion. 
EPJ Web of Conferences

\section{REFERENCES}

[1] Radebaugh R., Cryocoolers, part 2: Applications (Plenum Press), 1983, p. 129.

[2] Bowman R. C. and Scott Dowling R., Aerojet Rep., 9786 (1991) .

[3] Tegus O., Brǘck E., Buschow K. H. J. and De Boer F. R., Nature, 415 (2002) 150.

[4] Kawasaki H., Watanabe T. and Kanzawa A., Applied thermal engineering, 19 (1999) 133. 